\title{
Influence des bulles gazeuses et des préparations fermentatives complexes sur la viscosité des mélasses de betterave à sucre
}

\author{
Mahamat SEÏD ALI MALLOUM ${ }^{1 *}$, Abdelsalam TIDJANI ${ }^{1}$, Joseph DOSSOU ${ }^{2}$, \\ Mahamat Tahir ABAKAR ${ }^{3}$, Noel AKISSOE ${ }^{2}$ et Paulin AZOKPOTA ${ }^{2}$ \\ ${ }^{1}$ Université de N'Djamena, B.P.1117, N'Djamena, Tchad. \\ ${ }^{2}$ Université d'Abomey Calavi 01B.P526, Abomey Calavi, Benin. \\ ${ }^{3}$ Institut National Supérieur du Sahara et du Sahel d'Iriba, Iriba, Tchad. \\ *Auteur correspondant ; E-mail : seidaligarga@gmail.com; noel.akis@yahoo.fr; jokdossou@yahoo.fr; \\ Tel. : (00235)66 29 6716/(00229)97512024/(00229)97086266
}

\author{
REMERCIEMENTS \\ La présente étude a été financée par la Commission Nationale pour la Formation des Formateurs \\ (CONFOFOR).
}

\section{RESUME}

La mélasse est le coproduit de la fabrication du sucre de canne ou de betterave et dont la composition varie en fonction des conditions climatiques de production de la matière première et du processus de fabrication mis en place par la sucrerie. Elle se présente sous la forme d'un liquide épais, homogène, de couleur marron. Sa forte viscosité limite sa fluidité et son transport dans les tuyauteries et l'abaissement de cette viscosité par réchauffage engendre des coûts élevés d'énergie. L'objectif de cette étude est la mise en œuvre d'une méthodologie efficace permettant de diminuer la viscosité de la mélasse par l'usage des solutions liquéfiantes (ferments) sans toutefois augmenter la température. Cette diminution évitera à l'usine de dépenser énormément d'énergie pour baisser sa viscosité et faciliter sa fluidité dans les tuyauteries. Les travaux ont été réalisés en utilisant cinq (5) différentes préparations fermentatives liquides de masse volumique $1,2 \mathrm{~g} / \mathrm{cm}^{3}$ fabriquées dans une firme Danoise Novo-Nordisk et qui sont destinées à la transformation des produits céréaliers pour la production des boissons. Il s'agit notamment de : Ultraflo-L, Cereflo- 200L, Thermamyl120L, Fungamyl-800L et Ceremix- 6 XL. A travers cette recherche, il a été utilisé une mélasse, de pureté $55 \%$ laquelle, dans $100 \mathrm{~kg}$ de matière sèche retient $55 \mathrm{~kg}$ de saccharose et $45 \mathrm{~kg}$ de matières organiques et non organiques. Cette mélasse a été ensuite intensivement mélangée à l'aide d'un malaxeur mécanique, et périodiquement par intervalle d'un quart d'heure, des échantillons ont été prélevés et dont la masse volumique a été mesurée. Il ressort des expériences que les masses volumiques de ces échantillons diminuent respectivement en passant de 1,360 à 1,$320 ; 1,250$ et $1,200 \mathrm{~g} / \mathrm{cm}^{3}$. A la température constante de $60{ }^{\circ} \mathrm{C}$, maintenue à l'aide d'un ultra thermostat, la viscosité des échantillons a été mesurée. Les résultats obtenus ont montré que les viscosités finales de la mélasse de concentration $60 \%$ ont varié dans l'amplitude 10,5 à 15,3 m.Pa.s. Pour une thermo stabilité de la mêlasse avec un $\mathrm{pH}$ neutre des préparations fermentatives comme l'ultraflo- L et le cereflo -200L permettent de diminuer la viscosité de la mêlasse de 20-35\%.

(c) 2016 International Formulae Group. All rights reserved.

Mots clés : Mélasses, betterave, viscosité, température, ferments.

\section{Influence of gaz bubbles and complex fermentative preparation on the viscosity of the sugar beet molasses}

\section{ABSTRACT}

Molasses is the product of the manufacture of sugar cane or beet whose composition changes depending on the climatic conditions of de production of the raw materials and of the process of manufacture set up by the sugar refinery. It comes as a thick, homogenous, and brown liquid. Its fluidity is limited by its strong viscosity, 
hence the high cost of energy needed for its conveyance in the pipes, caused by the diminution of that viscosity through reheating. The aim of this study is the implementation of an efficient methodology allowing to lowering the viscosity of the molasses by the use of liquefying solutions without, however, increasing the temperature. This diminution will enable the factory not to use a lot of energy to lower the viscosity so as to ease fluidity in the pipes. The works have been carried out by using five (5) different liquid fermentative preparations of $1.2 \mathrm{~g} / \mathrm{cm}^{3}$ relative density made in a Danish Novo-Nordisk firm intended to the transformation of cereal products for the production of drinks. It is basically about: Ultraflo-L; Cereflo- 200L; Thermamyl120L; Ffungamyl-800L and Ceremix-6XL. In carrying out this scientific research, we have used the molasses of beet, of 55\% purity, which in $100 \mathrm{~g}$ of dried matters retain $55 \mathrm{~kg}$ of sucrose and $45 \mathrm{~kg}$ of organic and inorganic matters. This molasses has been then intensively mixed with a mechanic mixer, and periodically, at $15 \mathrm{mn}$ intervals, samples have been taken the relative density of which has been measured. It follows from our experiences that the relative densities of theses samples diminishes respectively, passing from 1.360 to 1.320 ; 1.250 and $1.200 \mathrm{~g} / \mathrm{cm}^{3}$. Densities of these samples taken respectively reduce from 1,$360 ; 1,320 ; 1,250 ; 1,200$ $\mathrm{g} / \mathrm{cm}^{3}$. At constant temperature of $60{ }^{\circ} \mathrm{C}$, Kept by an Ultra thermostat, the viscosity of samples have been measured. The results have shown that final viscosities of molasses of $60 \%$ concentration have varied from 10.5 to $15.3 \mathrm{~m}$. Pa in terms of amplitude. Thus, the thermo stability of the molasses with a neutral $\mathrm{pH}$ and a weak dose of fermentative preparations, like the Ultraflo-L and Cereflo-200L, allow the reduction of the viscosity of molasses to $20-35 \%$.

(c) 2016 International Formulae Group. All rights reserved.

Keywords: Molasses, beet, viscosity, temperature, ferments.

\section{INTRODUCTION}

La transformation de la canne à sucre ou de la betterave à sucre en sucre granulé, puis en sucre raffiné passe par un résidu appelé la mélasse contenant plus de $50 \%$ de saccharose. Elle constitue la plus importante matière première pour la fabrication de l'alcool éthylique, de levure et de l'acide citrique utilisé dans les boulangeries (Decloux, 2002). La mélasse de betterave se présente sous la forme d'un liquide visqueux et homogène de couleur marron. Elle est produite dans les régions tempérées, principalement en Europe. Le sucre, essentiellement le saccharose, est le composant le plus important de la mélasse de betterave (de 45 à 50\%). Elle contient aussi 10 à $12 \%$ de protéine brute dont 5 à $7 \%$ de bétaïne. Le sucre (saccharose) est produit à partir de la betterave sucrière ou de la canne à sucre. Ainsi, par exemple, la production mondiale, qui se chiffrait à 130,5 millions de tonnes en 2000-2001, se répartit pour environ $72 \%$ à partir de la canne à sucre et $28 \%$ à partir de la betterave. Dans les deux cas, du début de la filière à la production du sucre final, l'objectif des sucreries est de partir d'une matière première la plus pure possible et de produire, avec un rendement optimum, un sucre de qualité. La sucrerie est donc principalement une industrie de séparation et de purification. Un grand nombre d'opérations unitaires de séparation sont ainsi mises en œuvre (Decloux, 2002).

La mélasse contient une quantité considérable des matières organiques et minérales complexes comprenant des poly et oligosaccharides, des substances protéiques et des sels minéraux. Une bonne partie de la mélasse produite par les sucreries est utilisée pour la production du rhum industriel. Une autre fraction est utilisée dans l'alimentation des animaux et une petite partie se retrouve sur les tablettes des supermarchés pour la consommation humaine. La mélasse peut aussi être utilisée pour la culture des levures ainsi que, pour la production de divers produits, tels que l'acide acétique (vinaigre), l'acide citrique, le glycérol, l'acide aconitique, le glutamate, la lysine et l'éthanol (Patel et al., 2003).

La viscosité, ou résistance à l'écoulement, de n'importe quel fluide est une mesure primordiale du point de vue 
manipulation des fluides. Elle est une caractéristique du produit final et elle est très importante pour le contrôle du procédé. La viscosité des solutions de saccharose augmente de façon non linéaire avec la teneur en solides et diminue rapidement avec l'augmentation de la température. De façon générale, la viscosité augmente avec la diminution de la pureté du saccharose (Penning, 1990).

La mélasse est un sous-produit visqueux issu de la transformation de la canne à sucre ou de la betterave sucrière en sucre. $\mathrm{La}$ mélasse est une source d'énergie rapide et une source riche en minéraux. En comparaison à d'autres sources d'énergie, la teneur en calcium de la mélasse de canne est élevée (jusqu'à 1\%), alors qu'elle procure un faible apport en phosphore. La mélasse de betterave fournit un apport plus élevé en potassium et en sodium, mais un taux inférieur en calcium. La mélasse contient aussi des quantités importantes d'oligo-éléments, comme le cuivre (7 ppm), le zinc (10 ppm), le fer (200 ppm) et le manganèse (200 ppm). En raison de son aspect collant, la mélasse réduit la consistance poudreuse de certaines moulées finement broyées. Ainsi, la mélasse rend le mélange d'aliments plus savoureux pour le bétail (Homestead, 2015).

La mélasse étant une solution sursaturée, toutes les composantes se trouvent dans un état d'interactions formant des complexes et des agrégats qui ne se détruisent qu'à haute température (Orobinski et Serboulov, 1989). Selon ces mêmes auteurs, la dissolution de 55-65\% de mêlasse dans l'eau n'influe pas sur la structure de ces complexes (Orobinski, 1989). En Russie, plus de 250 millions de tonnes de mélasse sont transformés à cause de sa teneur élevée en matières sèches (Mahamat Seid, 1996). La viscosité de la mélasse non diluée de concentration $82 \%$ qui est de $6500 \mathrm{mPa}$.s à $20{ }^{\circ} \mathrm{C}$ (Tile et Languen, 1974) augmente plus de 15 fois $(91500 \mathrm{mPa} . \mathrm{s})$ lorsqu'on abaisse la température à $5{ }^{\circ} \mathrm{C}$.
En effet, pendant la période d'hiver, la fluidité de la mélasse est presque nulle, et les usines dépensent beaucoup d'énergie pour son chauffage et son déchargement. Cependant, lorsque l'on chauffe la mêlasse sa qualité se détériore. Par exemple, pour une température comprise entre $40-50{ }^{\circ} \mathrm{C}$, le pompage de la mêlasse n'est possible que par une pompe centrifuge spéciale (Sapronov, 1986). Ainsi, augmenter la fluidité de la mélasse sans la réchauffer est une question d'actualité, économiquement rationnelle. La viscosité, ou résistance à l'écoulement, de n'importe quel fluide est une mesure primordiale du point de vue manipulation des fluides. Elle est une caractéristique du produit final et elle est très importante pour le contrôle du procédé. La viscosité des solutions de saccharose augmente de façon non linéaire avec la teneur en solides et diminue rapidement avec l'augmentation de la température (Alfa et al., 2012). Il est aussi important de valoriser la mélasse car le saccharose y cesse de cristalliser parce que la viscosité augmente lorsqu'on démunie sa pureté. Cela empêche la molécule de se déplacer et de se déposer sur la surface du cristal. Par ailleurs, chacun des composés chimiques dissous dans la mélasse va séquestrer une partie du sucre et l'empêcher de cristalliser, d'où l'appellation d'effet melassigène attribuée aux non-sucres de la mélasse (Mathlouthi et al., 2012).

De façon générale, la viscosité augmente avec la diminution de la pureté du saccharose. A l'état liquide, les produits sucriers, y compris la mélasse, contiennent des bulles gazeuses (l'air, $\mathrm{CO}_{2}$ et autres) dont l'existence retarde le processus technologique. Ces gaz se regroupent dans l'air pendant les processus d'agitation et mélange de solution de sucre et apparaissent soit au cours de la réaction conduisant à la formation de mélanoïdine, soit pendant le largage d'une partie de sucre ou lorsque le produit est couvert de la mousse. La taille des bulles dépend essentiellement de deux facteurs à savoir la quantité de $\mathrm{CO}_{2}$ et de la hauteur du récipient devant le contenir. Moins il y a de 
$\mathrm{CO}_{2}$ plus les bulles sont fines (Liger-Belair et al., 2010).

\section{MATERIEL ET METHODES}

Matériel

Mélasse

La mélasse de pureté $55 \%$ en provenance de l'usine sucrière de Moscou a été obtenue en traitant le sucre brut de betterave de concentration $81,5 \%$. Puis elle a été diluée jusqu' à une concentration de $60 \%$ en matières sèches.

\section{Solutions liquéfiantes (Ferments)}

Quatre différentes préparations fermentatives liquides de couleur brun sombre et de masse volumique $1,2 \mathrm{~g} / \mathrm{cm}^{3}$ ayant été fabriquées dans une firme Danoise NovoNordisk pour la production de la bière, ont été utilisées pour les expériences. Il s'agit de :

- Ultraflo-L contenant du $\beta$-glucosane (pentosane) et de la cellulase extrait du champignon Humicola isolens; cet ferment a été utilisé à la concentration de $0,28 \mathrm{~g} / \mathrm{kg}$ de mélasse ;

- Cereflo -200L contenant de l'endo- $\beta$ glucosane, d'origine bactérienne (Bacilius Subtilis), utilisé à la concentration de 1,9 $\mathrm{g} / \mathrm{kg}$ de mélasse ;

- Thermamyl -120L contenant de l' $\alpha$ amylase stable, à températures variant entre 80 et $120{ }^{\circ} \mathrm{C}$, d'origine bactérienne (Bacillus licheniformis) utilisé à la concentration de $0,6 \mathrm{~g} / \mathrm{kg}$ de mélasse ;

- Fungamyl-800 L contenant de l' $\alpha$ amylase provenant $\mathrm{du}$ champignon Aspergillus oryzae, a été utilisé à la concentration de $0,35 \mathrm{~g} / \mathrm{kg}$ de mélasse.

Outre ces quatre ferments, un cinquième complexe, d'origine bactérienne (Bacilus subtilus), le Ceremix -6 XL, de forme granulée et de couleur brun sombre contenant de l' $\alpha$-amylase, du protéinase et de l' $\beta$ - glucosane, agissant sur la gélatine semisucrée, a été utilisée à la concentration de 0,9 $\mathrm{g} / \mathrm{kg}$ de mélasse dans le même but que les autres ci-dessus précités.

\section{Méthodes}

Au début des expériences, la viscosité initiale de la mélasse était de $15,8 \mathrm{mPa}$.s pour une température de $60{ }^{\circ} \mathrm{C}$ et la durée de la réaction était de $90 \mathrm{mn}$. A la fin de la réaction, pour activer les ferments et mesurer la viscosité, la mélasse a été d'abord chauffée à $100{ }^{\circ} \mathrm{C}$ puis refroidie jusqu'à $60{ }^{\circ} \mathrm{C}$. La mélasse a été diluée à $60 \%$, puis ensemencée de chaque ferment conformément à la température de $60^{\circ} \mathrm{C}$ et au $\mathrm{pH} \quad 6-7$ recommandés par l'usine fabricante. La mélasse a été ensuite intensivement mélangée avec le malaxeur mécanique, et périodiquement, des échantillons ont été prélevés pour la mesure de la masse volumique. En augmentant la durée de mélange, la mélasse devient de plus en plus saturée de bulles d'air. La solution de chaque batch a été ensuite thermo stabilisée pendant $90 \mathrm{mn}$ par agitation. Pour chaque batch, les échantillons ont été prélevés de manière périodique toutes les15 minutes. Chaque échantillon a été d'abord mis dans un thermostat sous une dépression qui varie entre 46,66.10 $-53,32 \cdot 10^{6} \mathrm{~Pa}$.

A température constante de $60{ }^{\circ} \mathrm{C}$, maintenue à l'aide d'un ultra thermostat, la viscosité des échantillons a été mesurée grâce au viscosimètre de Guepler à chute des billes.

Les autres séries des analyses ont été réalisées avec des différentes concentrations des préparations fermentatives pendant 90 $\mathrm{mn}$, à une température de $60^{\circ} \mathrm{C}$.

\section{RESULTATS}

Les paramètres de la mélasse (masse volumique, volume d'air et viscosité) issue des solutions liquéfiantes sont présentés dans le Tableau 1.

Les caractéristiques des solutions de mélasses ont varié en fonction des ferments (solutions liquéfiantes). Le volume d'air était dans l'amplitude $0-11,7 \mathrm{~cm}^{3}$, avec la plus forte valeur obtenue par Fungamyl-800L et la plus faible par Ultraflo-L (Tableau 1). La viscosité a évolué dans le même sens tandis que la masse volumique avait une tendance opposée. 
Une forte corrélation positive et significative $\left(R^{2}=0,999\right)$ a été observée entre le volume de l'air et la viscosité des solutions de mélasse. Des préparations fermentatives : 1-Ultraflo-L; 2-Cereflo-200L ; 3-Ceremix -6XL ; 4Termamyl-120L ; 5-Fungamyl-800Lont été utilisées.

La Figure 2 montre l'évolution de la viscosité sous l'action de ferment. Indépendamment des ferments, une chute de la viscosité a été observée mais elle était plus marquée avec l'ultraflo-L qui est apparu comme le ferment le plus actif. En effet, l'ultraflo-L a réduit la viscosité de la mêlasse de plus d'un tiers en une heure de traitement (une baisse de $32 \%$ après $60 \mathrm{mn}$ ) alors que cette performance n'est atteinte par aucun des autres ferments durant la période de réaction (90 mn).

Une relation négative a été observée entre les concentrations des ferments et la viscosité de la mêlasse.

Par ailleurs, la chute de la courbe a été plus remarquable au niveau de ferment Utraflo-L que les autres ferments.

Dans le Tableau 3 sont représentés les résultats de la détermination des conditions optimales des effets des trois ferments les plus actifs sur la base des paramètres recommandés par l'usine de fabrication. Les viscosités finales de la mélasse de concentration $60 \%$ sous l'effet de préparation fermentative, traitée dans les conditions indiquées dans le Tableau 2 pendant une durée de 90 minutes, ont varié dans l'amplitude 10,5 à 15,3 mPa.s. Le Fungamyl-800 a donné la plus forte valeur et l'Ultraflo-L la plus faible (Tableau 3).

Il ressort des résultats obtenus (Tableaux 2, 3 et 4) que les préparations fermentatives qui ont un effet considérable sur la diminution de la viscosité sont-Ultraflo-L contenant du $\beta$-glucosane; le Cereflo-200L contenant de l'endo- $\beta$-glucosane, d'origine bactérienne (Bacilius subtilis) et le Ceremix -6 XL.

Cette action sélective est conditionnée par la composition des non-sucres qui proviennent de la mélasse et qui se sont formés pendant le processus de production. Un $\mathrm{pH}$ neutre, une basse température et une faible dose des préparations fermentatives comme l'ultraflo-L et le cereflo-200L, permettent de diminuer la viscosité de la mélasse de $20-35 \%$.

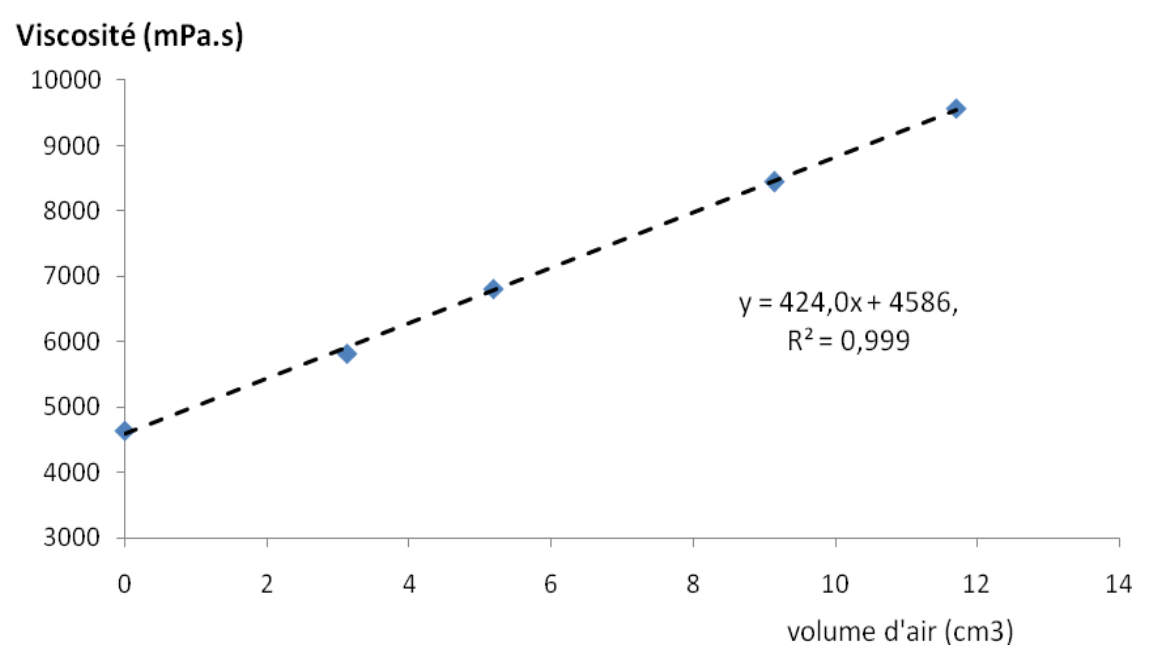

Figure 1 : Relation entre le volume de l'air et la viscosité des solutions de mélasse. 


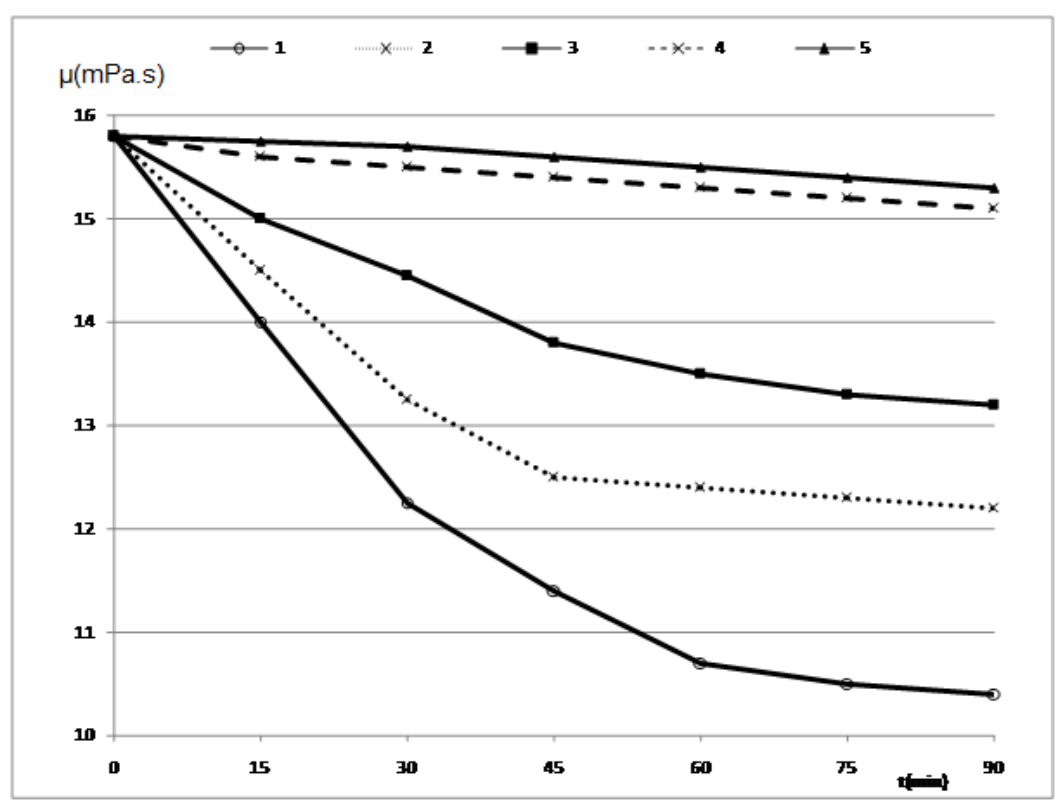

Figure 2 : Variation de la viscosité de solution des mélasses de concentration $60 \%$, sous l'effet du ferment.

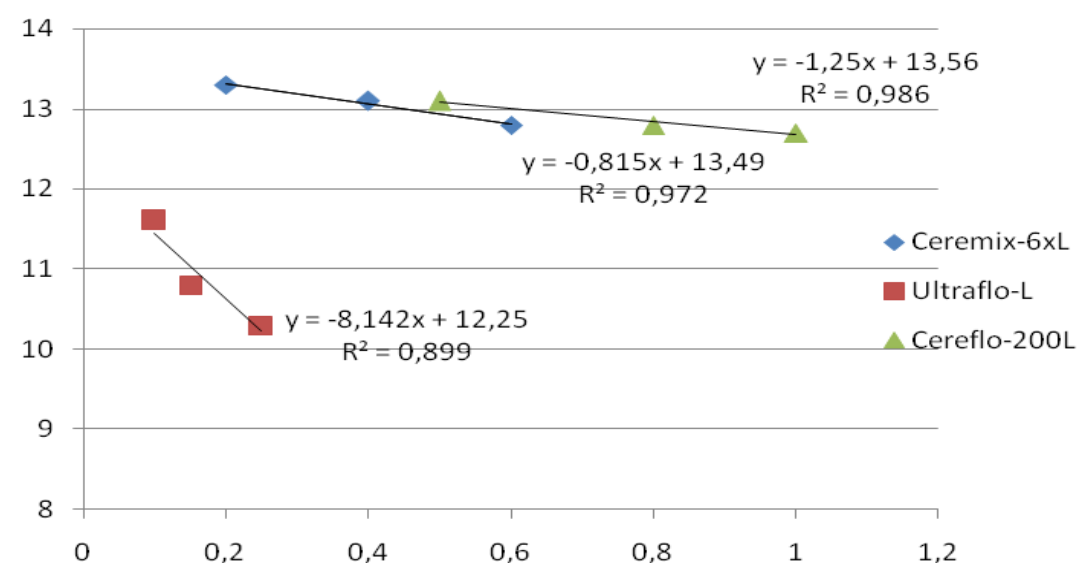

Figure 3 : Variation de la viscosité de mélasse en fonction de la concentration des ferments.

Tableau 1 : Effet de la durée de la réaction de 90 mn sur les caractéristiques des solutions de mélasses.

\begin{tabular}{lcccc}
\hline Echantillons & $\begin{array}{c}\text { Masse volumique } \\
\left(\mathbf{g} / \mathbf{c m}^{\mathbf{3}}\right)\end{array}$ & $\begin{array}{c}\text { Rapport } \\
\left(\mathbf{\rho} / \mathbf{\rho}_{\mathbf{t}}\right)\end{array}$ & Volume de l'air, $\mathbf{( \mathbf { c m } ^ { 3 } )}$ & $\begin{array}{c}\text { Viscosité } \\
(\mathbf{m P a . s})\end{array}$ \\
\hline 1- Ultraflo-L & 1,425 & 1,00 & 0 & 4640 \\
2-Cereflo-200L & 1,360 & 1,05 & 3,12 & 5820 \\
3-Ceremix -6XL & 1,320 & 1,07 & 5,19 & 6815 \\
4 -Termamyl-120L & 1,250 & 1,14 & 9,14 & 8450 \\
5 -Fungamyl-800L & 1,200 & 1,18 & 11,70 & 9570 \\
\hline
\end{tabular}


Tableau 2 : Influence de la concentration en ferment sur la viscosité de mélasse de concentration $60 \%$ en matière sèche, thermo stabilisée pendant $90 \mathrm{mn}$ sous $60{ }^{\circ} \mathrm{C}$.

\begin{tabular}{lccc}
\hline Préparation fermentative & $\begin{array}{c}\text { Concentration en } \\
\text { ferment de mélasse, } \mathbf{c m}^{\mathbf{3}}\end{array}$ & Viscosité \% & diminution de la viscosité en \% $\mathbf{~ d a n s ~ 1 0 0 0 ~} \mathbf{~ c m}^{\mathbf{3}}$ \\
\hline \multirow{2}{*}{ Ultraflo-L } & 0,1 & 11,6 & 26,4 \\
& 0,15 & 10,8 & 31,7 \\
& 0,25 & 10,3 & 34,7 \\
Cereflo-200L & 0,5 & 13,1 & 17,2 \\
& 0,8 & 12,8 & 18,9 \\
\hline \multirow{2}{*}{ Ceremix-6XL } & 1,0 & 12,7 & 19,6 \\
& 0,2 & 13,3 & 17,6 \\
\hline
\end{tabular}

Tableau 3 : Diminution de la viscosité de mélasse de concentration 60\% sous l'effet de préparation fermentative, pendant 90 min.

\begin{tabular}{|c|c|c|c|c|c|c|c|c|}
\hline & \multicolumn{4}{|c|}{ Condition de l'interaction } & \multirow{2}{*}{\multicolumn{2}{|c|}{$\begin{array}{l}\text { Teneur en ferments dans } \\
1 \text { kg des matières sèches }\end{array}$}} & \multirow{2}{*}{\multicolumn{2}{|c|}{$\begin{array}{c}\text { Viscosité à } 60{ }^{\circ} \mathrm{C}, \\
\text { mPa.s }\end{array}$}} \\
\hline & \multicolumn{2}{|c|}{ pH } & \multicolumn{2}{|c|}{ Température $\left({ }^{\circ} \mathbf{C}\right)$} & & & & \\
\hline & $\begin{array}{c}\text { Recommandé } \\
\text { par l'usine }\end{array}$ & Expérimental & $\begin{array}{l}\text { Recommandé } \\
\text { par l'usine }\end{array}$ & Expérimentale & $\begin{array}{c}\text { Recommandé } \\
\text { (unité) } \\
\text { par l'usine }\end{array}$ & Expérimentale & Initiale & Finale \\
\hline Ultraflo-L & $5-7$ & 7 & $55-70$ & 60 & $0,20-0,51$ & 0,28 & 15,8 & 10,5 \\
\hline Cereflo-200L & $6-7$ & 6 & 68 & 60 & $1,93-2,84$ & 1,4 & 15,8 & 12,3 \\
\hline Ceremix-6XL & $5,7-6$ & 6 & $45-50$ & 50 & 0,6 & 0,72 & 15,8 & 13,1 \\
\hline Termamyl-120 & 6 & 6 & 90 & 90 & $0,3-0,5$ & 0,35 & 15,8 & 15,1 \\
\hline Fungamyl -800 & 5 & 5 & 5,5 & 50 & $0,5-1$ & 0,2 & 15,8 & 15,3 \\
\hline
\end{tabular}




\section{DISCUSSION}

Considérant que les ferments utilisés sont destinés à la destruction des autres sucres et autres polysaccharides (amidon) de grande liaison moléculaire et sachant que leur pourcentage dans la mélasse est insignifiante (Goza-Leon et Perez de Alejo, 2000), les résultats issus de ces travaux ont montré qu'il est possible de diminuer la concentration des préparations fermentatives pendant le traitement de la mélasse.

Des travaux dont le but est de proposer des stratégies pour réduire la consommation d'énergie et les pertes lors du procédé sont réalisés pour des usines spécifiques (Wright, Silva et Amador, 2005; Lavarack et al., 2004). Ces travaux apportent des solutions claires et applicables à l'usine analysée, lui permettant ainsi d'améliorer sa productivité et sa rentabilité.

Dans l'objectif de maîtriser le processus de production et améliorer, par conséquent, la qualité du produit fini, des études scientifiques des points de vue physico-chimique, microbiologique et organoleptique ont été entreprises. Ce procédé est basé principalement sur l'utilisation de ferments lactiques sélectionnés (Lactobacillus plantarum S175 et Lactobacillus pentosus S100). Les résultats ont montré une amélioration nette du procédé de transformation en termes de durée du processus de fermentation du taux d'altération des olives et de la qualité organoleptique du produit fini (Abelsalam Asehraou et al., 2014).

Pour l'utilisation pratique, il est recommandé d'utiliser les proportions des préparations fermentatives de la firme Danoise (NOVO Nordiks) dans les conditions optimales de leurs effets cidessous indiquées :

Pour le ferment Ultraflo-L, une quantité de $0,2 \mathrm{~cm}^{3}$ pour $1 \mathrm{~kg}$ de matières sèches dans la mélasse, avec un pH 6-7 et une température de $60{ }^{\circ} \mathrm{C}$;

Par ailleurs, pour le Cereflo-200L et le Ceremix-6XL respectivement il est souhaitable de prendre une quantité de $0,8 \mathrm{~cm}^{3}$ pour $1 \mathrm{~kg}$ des matières sèches dans la mélasse avec un pH 6-7 et une température de $60{ }^{\circ} \mathrm{C}$ et $0,6 \mathrm{~cm}^{3}$ pour $1 \mathrm{~kg}$ de mélasse, avec un $\mathrm{pH} 5$, 7-6 et une température de $50^{\circ} \mathrm{C}$.

\section{Conclusion}

$\mathrm{Au}$ vu des résultats des expériences réalisées, il a été établi que certains ferments complexes ont une influence considérable sur la baisse de la viscosité de mélasse. Ainsi donc, pour une thermo stabilité de la mélasse avec un $\mathrm{pH}$ neutre et une faible dose, les préparations fermentatives comme l'ultraflo$\mathrm{L}$ et le cereflo-200L, ont permis de diminuer la viscosité de la mélasse de 20-35\%.

\section{CONFLIT D'INTERETS}

Les auteurs déclarent qu'ils n'ont aucun conflit d'intérêts.

\section{REMERCIEMENTS}

La présente étude a été appuyée techniquement par le Centre National de la Recherche pour le Développement (CNRD) du Ministère de l'Enseignement Supérieur et de la Recherche Scientifique du Tchad. Nous remercions vivement tous ceux qui ont contribué directement ou indirectement à la réalisation de ce travail scientifique et plus particulièrement au Professeur SAPRONOV ALEKCEI ROMANOVITCH, mon Directeur de thèse pour son appui scientifique.

Qu'ils trouvent ici toute ma reconnaissance et ma gratitude.

\section{REFERENCES}

Abdeslam A, Yahya RP, Nabil GP, Zahra LP, Philipe AA, d'Arthabaska S-N. 2012. Extraction et Raffinage du sucre de canne, $67 \mathrm{p}$.

Goza-Leon O, Perez De Alejo VH. 2000. An Expert System for the Analysis of Energy Systems in cane Sugar Factories. New Review of Applied Expert Systems and Emerging Technologies, 6: 65-80. 
Homestead O. 2015. Journal de Homestead Organics (google) Instruction technologique TI 10-05031531-102995 (anonyme).

Lavarack BP, Hodson JJ, Broadfootr R, Vigh S, Venning J. 2004. Improving the Energy Efficiency of Sugar Factories: case Study for Pioneer Mill. International Sugar Journal, 106(1266): 337-343.

Liger-Belair G, Gougeon R, SchmittKopplin Ph. 2010. Champagne: l'arôme au cœur des bulles. Pour la Science, 398 : 34-38.

Mahamat SA. 1996. Thèse de Doctorat: Perfectionnement de la Technologie de traitement et de Conservation de Sucre brut, Moscou

Martine Decloux. 2002. Procédés de transformation en sucrerie. Référence F6150.

Mathloulti MC, Barbara R. 2012. Extraction de sucre. CEDU avec la collaboration de l'Université de Reims, 47p.

Orobinski IP, Serboulov Boldirov AP. 1989. Optimisation de la viscosité des solutions sucrières en présence de certains non sucres pour des températures $30{ }^{\circ} \mathrm{C}$ et $40{ }^{\circ} \mathrm{C}$. Institut Technologique de Voronej $\mathrm{N}^{\circ} 1977$.

Patel RM, Desai AJ. 2003. Biosurfactant production by Pseudomonas aeruginosaGS3 from molasses. Online. Letters in Applied Microbiology, 25(2): 91-94. DOI: $10.1046 /$ j.1472765X.1997.00172.x;

Penning, Baker. 1990. Effet de la concentration et de la température sur la viscosité de saccharose.

Sapronov AR. 1986. Technologie de production de sucre, $431 \mathrm{p}$.

Tile X, Languen A. 1974. La viscosité des solutions sucrières. International Sugar Journal, 906: 169-173.

Wright PG, Silva TA, Amador K. 2005. Improving the Process Steam economy in a Cane Sugar Factory: A Case History of A Successful Cogeneration Project. International Sugar Journal, 107(1279): 425-430. 